\title{
Astrophysical Jets: A Review
}

\author{
J. H. Beall*i \\ Space Sciences Division, NRL \& St. John's College, USA \\ E-mail: beallesjc.edu
}

\begin{abstract}
Astrophysical jets can be defined most generally as linear structures observed in the sky. These jets are typically associated with accretion processes in star forming regions, compact objects (such as neutron stars and black holes), $\gamma$-ray bursts, and in some pulsars. The jets can be extremely powerful in terms both of their kinetic luminosity and radiative signatures, and often emit radiation over the entire electromagnetic spectrum, from radio to $\gamma$-rays. Astrophysical jets often appear to be one-sided and Doppler boosted. This phenomenon is usually interpreted as evidence of bulk relativistic motion of the emitting particles. In the last three decades and more and as a result of observing campaigns using detectors sensitive from radio to $\gamma$-ray energies, theoretical models of these sources have become richer and more complex. They have moved from assumptions of isotropy that made analytic calculations possible, to fully anisotropic models of emission from the jets and their interactions with the interstellar and intra-cluster medium. Such anisotropic calculations are only possible because we now have extensive computational resources. In addition, the degree of international cooperation required for observing campaigns of these sorts is remarkable, since the instruments include among others the Very Large Array (VLA), the Very Long Baseline Array (VLBA), and entire constellations of satellite instruments, often working in concert. In this paper, I discuss some relevant observations from these efforts and the theoretical interpretations they have occasioned. keywords: astrophysical jets, active galactic nuclei, UHE cosmic rays, quasars, microquasars
\end{abstract}

Frontier Research in Astrophysics

26 - 31 May, 2014

Mondello (Palermo), Italy

* Speaker.

${ }^{\dagger}$ A footnote may follow. 


\section{Introduction}

Extended linear structures, usually interpreted as "astrophysical jets," are found in star-forming regions, in compact binaries, and of course in active galactic nuclei (AGNs), and galaxy clusters. $\gamma$-ray bursts have been associated with astrophysical jets formed in stellar core collapse of massive stars, where the jets is directed toward Earth. We have become aware of considerable details of these results through decades of sustained observing campaigns.

The results of these campaigns show similarities and significant differences in the data from some epochs of galactic microquasars, including GRS 1915+105, the concurrent radio and x-ray data (Beall et al., 1978) on Centaurus A (NGC 5128), 3C120 (Marscher (2006), and 3C454.3 as reported by Bonning et al. (2009), to name a few. The Bonning et al. analysis of the $3 \mathrm{C} 454.3$ data showed the first results from the Fermi LAT Space Telescope for the concurrent variability at optical, UV, IR, and $\gamma$-ray variability of that source. In combination with observations of microquasars and quasars from the MOJAVE Collaboration (see, e.g., Lister et al., 2009), these observing campaigns provide an understanding of the time-dependent evolution of these sources at milliarcsecond resolutions (i.e., parsec for AGNs, and Astronomical Unit scales for microquasars).

In blazar sources (see, e.g., Ulrich et al. 1997, and Marscher, 2006) there seems to be a confirmed connection of jets with accretion disks. In sources without large-scale linear structures (i.e., jets), as Ulrich et al. (1997) note, the source variability could result from the complex interactions of the accretion disk with an x-ray emitting corona. But to the extent that "small" jets are present in these sources, the disk-jet interaction must still be of paramount importance, since it provides a mechanism for carrying away energy from the disk.

Current theories (see e.g., Hawley 2003 and Bisnovatyi-Kogan 2012 for a discussion of disk structure and jet-launch issues, respectively) suppose that the jet is formed and accelerated in the accretion disk. But even if this is true in all sources, it is still unclear whether or not astrophysical jets with shorter propagation lengths are essentially different in constitution from those that have much longer ranges, or whether the material through with the jet propagates determines the extent of the observational structures we call jets. At all events, the complexities of the jet-ambient medium interaction must have a great deal to do with the ultimate size of emitting region.

This sort of argument has applications to both quasars and microquasars, especially if essentially similar physical processes occurs in all these objects (see, e.g., Beall, 2003, and Marscher, 2006). To some, it has become plausible that essentially the same physics is working over a broad range of temporal, spatial, and luminosity scales. Hannikainen (2008) and Chaty (2007) have discussed some of the emission characteristics of microquasars, and Paredes (2007) has considered the role of microquasars and AGNs as sources of high energy $\gamma$-ray emission. In fact, the early reports of the concurrent radio and $\mathrm{x}$-ray variability of Centaurus A can be plausibly interpreted as the launch of a jet from Cen A's central source into the complex structures in its core. Additionally, these observations are remarkably similar to the observations of galactic microquasars and AGNs, including the observations from the Fermi LAT Space Telescope of concurrent $\gamma$-ray, IR, optical, and UV variability of 3C454.3 (Bonning et al., 2009), and observations by Madejski et al. (1997) for BL Lac, among others. 


\section{Concurrent Variability of AGN Jet Sources as an Indication of Jet Launch or Jet-Cloud Interactions}

As examples, we now turn to two such observations in this paper, the concurrent radio and $\mathrm{x}$ ray variability of Centaurus A (Beall et al., 1978), and the $\gamma$-ray, UV, and IR concurrent variability discussed by Bonning et al., (2009) using the Fermi LAT and Swift spacecraft.

\subsection{Radio and X-ray Variability Measurements of Centaurus A (NGC 5128)}

The first detection of concurrent, multifrequency variability of an AGN came from observations of Centaurus A (Beall et al., 1978, see Figure 1 of that paper). Beall et al. conducted the observing campaign of Cen $\mathrm{A}$ at three different radio frequencies in conjunction with observations from two different $\mathrm{x}$-ray instruments on the OSO-8 spacecraft in the $2-6 \mathrm{keV}$ and $100 \mathrm{keV} \mathrm{x}$-ray ranges. These data were obtained over a period of a few weeks, with the Stanford Interferometer at $10.7 \mathrm{GHz}$ obtaining the most data. Beall et al. also used data from other epochs to construct a decade-long radio and x-ray light curve of the source. Figure 1a of Beall (2011) shows the radio data during the interval of the OSO-8 $\mathrm{x}$-ray observations, as well as the much longer timescale flaring behavior evident in the three different radio frequencies and at both low-energy (2-6 keV, see Figure 1b of Beall, 2011) and in high-energy ( $100 \mathrm{keV}$, see Figure 1c of Beall, 2011) x-rays. As noted by Beall (2011), a perusal of Figure 1a in that paper shows that the radio data (represented as a " + " in that figure) generally rise during 1973 to reach a peak in mid-1974, then decline to a relative minimum in mid-1975, only to go through a second peak toward the end of 1975, and a subsequent decline toward the end of 1976. This pattern of behavior is also shown in the $\sim 30 \mathrm{GHz}$ data and the $\sim 90 \mathrm{GHz}$ data albeit with less coverage at the higher two radio frequencies.

Several points are worthy of note. First, as Beall et al. (1978) show, the radio and x-ray light curves track one another. This result demonstrated the first report of concurrent radio and x-ray variability of an active galaxy. Mushotzky et al. (1978), using the weekly $10.7 \mathrm{GHz}$ data obtained by Beall et al., (1978) demonstrate that the $10.7 \mathrm{GHz}$ radio data track the 2-6 keV x-ray data on weekly time scales, also. The concurrent variability at radio and $\mathrm{x}$-ray frequencies argues that the emitting region is the same for both the radio and $\mathrm{x}$-ray light. This, as was noted by Beall and Rose (1980), can be used to set interesting limits on the parameters of the emitting region. In addition, the observations at the three radio frequencies $(10.7 \mathrm{GHz}, \sim 30 \mathrm{GHz}$, and $\sim 90 \mathrm{GHz})$ clearly track one another throughout the interval whenever concurrent data are available.

A plausible hypothesis for the observations we have witnessed is that they arise from physical processes associated with the "launch" of an astrophysical jet into the complex structures in the core of Centaurus A. The timescale of the evolution from early 1973 through 1977 appears to be associated with the evolution of a larger structure over a more extended region. The observations are consistent with the interaction of the astrophysical jet with an interstellar cloud in the core of Cen A.

It is clear from this discussion that a distinction needs to be made about which observational signatures are associated with the jet launch, the jet itself, and the ambient medium's reaction to the jet. In considering such a scenario applied to microquasars, the observations of Sco X-1 by Fomalont, Geldzahler, and Bradshaw (2001), as discussed by Beall et al. (2013) are extremely informative. 


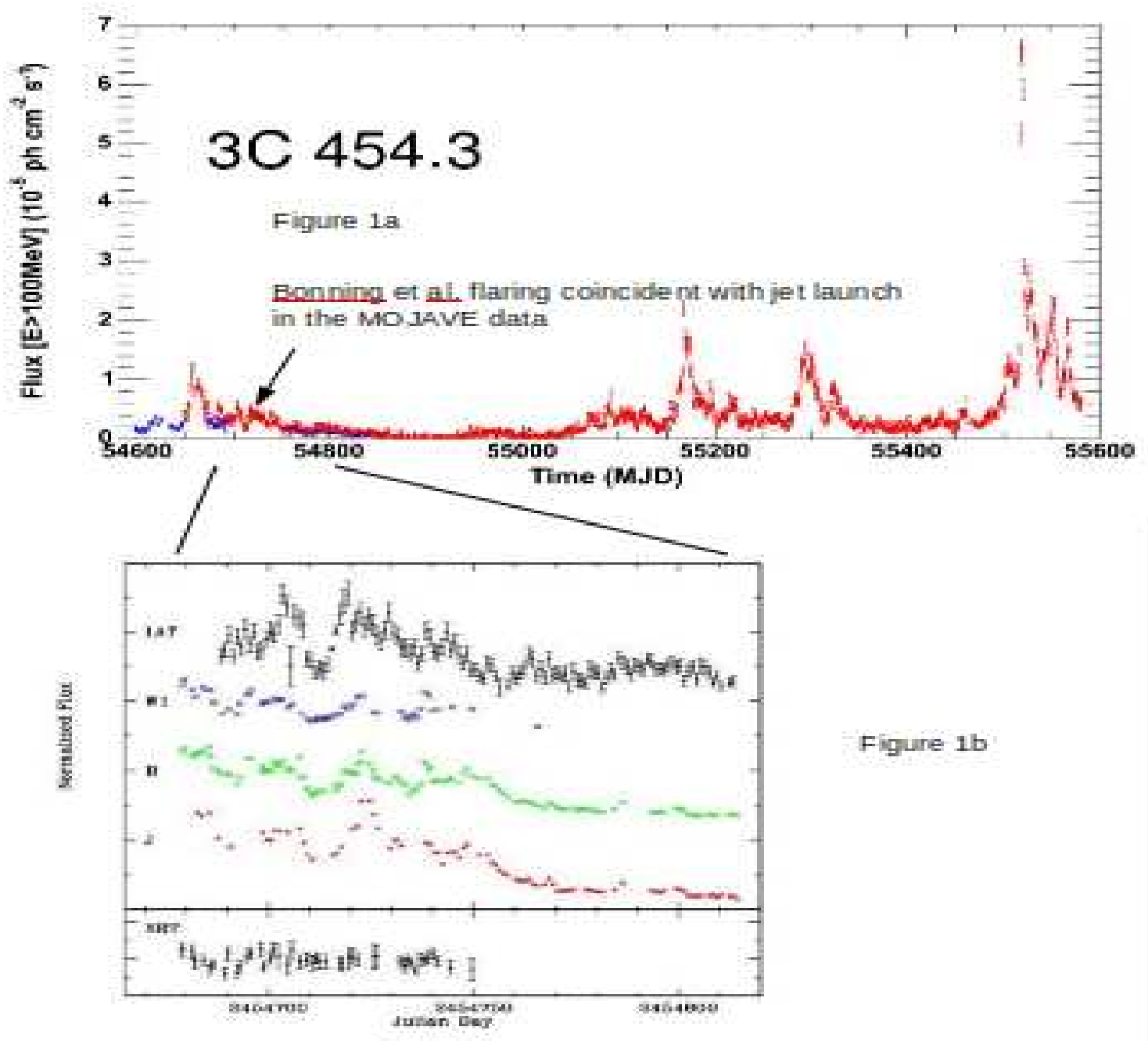

Figure 1: Fermi LAT data (see, e.g., Lott et al. 2013) showing the time history of the flaring from 3C454.3 (Figure 1b), along with the Bonning et al. (2009) data showing concurrent $\gamma$-ray, IR, optical, and UV data (Figure 1b). The time of the jet launch from the MOJAVE "movie" is illustrated by the arrow. The result, first reported here, shows radio flaring consistent with the Bonning et al. data.

\subsection{A Jet Launch Coincident with the 3C454.3 Multi-frequency Flaring}

Bonning et al. (2009) performed an analysis of the multi-wavelength data from the blazar, 3C454.3, using IR and optical observations from the SMARTS telescopes, optical, UV and X-ray data from the Swift satellite, and public-release $\gamma$-ray data from the Fermi LAT experiment. In that work, she demonstrated an excellent correlation between the IR, optical, UV and $\gamma$-ray light curves, with a time lag of less than one day.

Urry (2011) noted that 3C454.3 can be a laboratory for multifrequency variability in Blazars. 
While a more precise analysis of the data will be required to determine the characteristics of the emitting regions for the observed concurrent flaring at the different frequencies, the pattern of a correlation between low-energy and higher-energy variability is consistent with that observed for Cen A, albeit with the proviso that the energetics of the radiating particles in $3 \mathrm{C} 454.3$ is considerably greater.

A perusal of the data for 3C454.3 at milliarcsecond scales taken from the MOJAVE VLBA campaign during the Bonning et al (2009) flare show that the time-dependent flare occurs during launch of a new component of the jet that originates from the core.

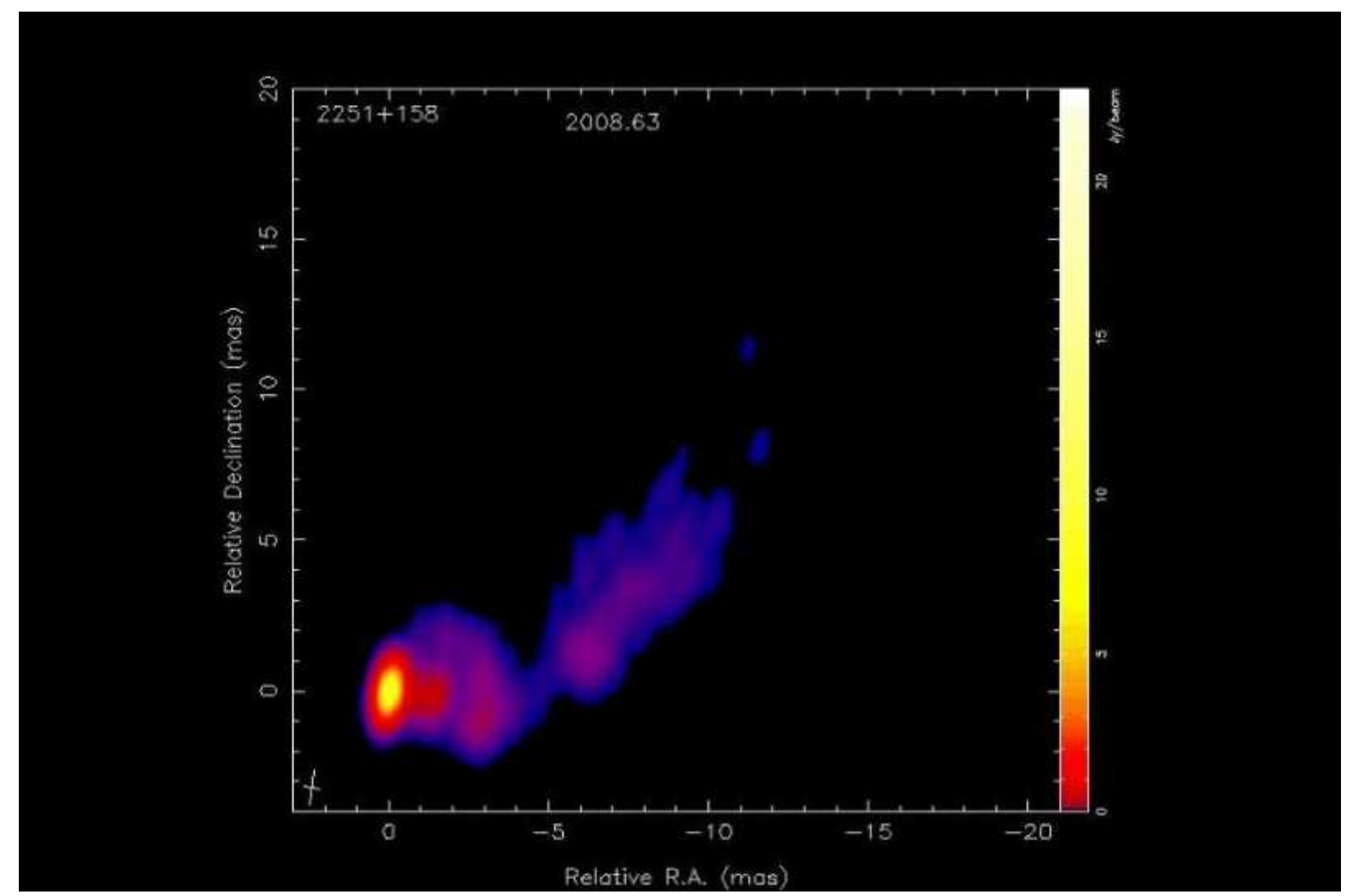

Figure 2: Figure 2: 3C454.3 shown at milliarcsecond scales for data taken from the MOJAVE VLBA campaign during the Bonning et al (2009) flare. A perusal of the time-dependent flare clearly shows that the jet launch from the core of the AGN is coincident with the double peaked flaring shown in the Bonning et al. results.

The pattern of variability reported by Bonning et al., is consistent with the injection of relativistic particles into a region with relatively high particle and radiation densities (i.e., an interstellar cloud). The picture that emerges, therefore, is consistent with the observations of spatially and temporally resolved galactic microquasars and AGN jets.

It should not escape our notice that other epochs in the 3C454.3 data from MOJAVE and Fermi LAT also show similar periods of injection of radio blobs that are associated with a double-peaked structure in the $\gamma$-ray light. The Fermi LAT data (see, e.g., Lott et al. 2013) show that the time history of the flaring from $3 \mathrm{C} 454.3$ are associated with times for several additional flares in the MOJAVE data sets (see Figure 3). The flares are similar to those shown in Figure 2, and clearly indicate the correlation of $\gamma$-ray flares with the jet launches. These associations have also been 
pointed out by Jorstadt et al. 2013, who provide a detailed review of three outbursts in their paper. The direct correlation of the Fermi LAT and the MOJAVE data can clearly be seen in the figure. The data show evidence for the repeated launching of the astrophysical jets into the ambient medium. The jet structure is apparently not a continuous injection over long time scales, but an injection of a series of "blobs" that are co-aligned along some preferred axis.

\section{Additional MOJAVE jet launches}

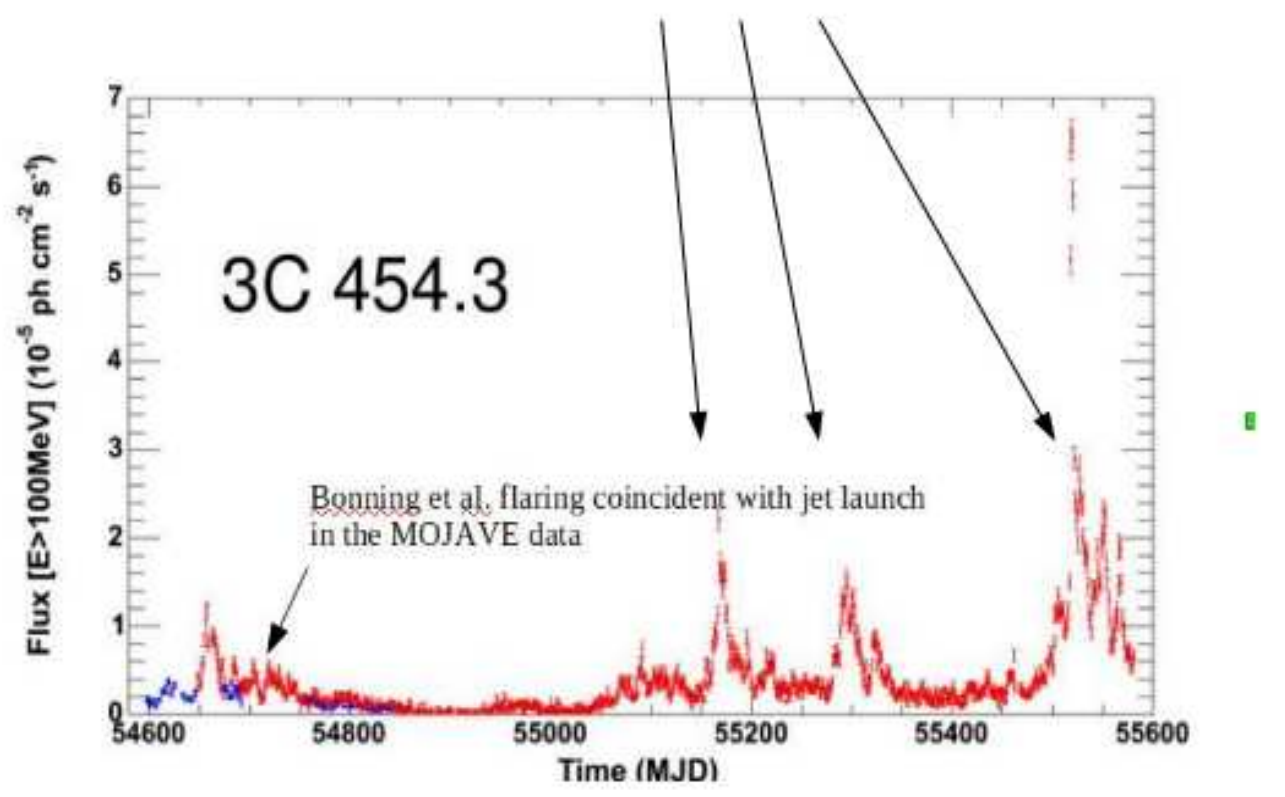

Figure 3: Fermi LAT data (see, e.g., Lott et al. 2013) showing the time history of the flaring from 3C454.3 along with times for several additional flares in the MOJAVE data sets, The flares are similar to those shown in Figure 2 above, and clearly indicate the correlation of $\gamma$-ray flares with the jet launches. These associations have also been pointed out by Jorstadt et al. 2013, who provide a detailed review of three outbursts in their paper. The direct correlation of the Fermi LAT and the MOJAVE data can clearly be seen in the figure. The data show evidence for the repeated launching of the astrophysical jets.

For the Cen A data, and for data from 3C454.3, it is the concurrent variability that suggests that the radio to x-ray (in Cen A's case) and the IR, optical, and UV to $\gamma$-ray fluxes (in 3C454.3's case) are created in the same region. This leads to the possibility of estimates of the source parameters that are obtained from models of these sources. VLBI observations of cores vs. jets (see, e.g., the study of BL Lac by Bach et al. 2006) show the structures of the core vs. jet as they change in frequency and time. It has thus become possible to separate and study the time variability of the jet and the core of AGN at remarkably fine temporal and spatial scales.

Van der Laan (1966) discussed the theoretical interpretation of cosmic radio data by assuming a source which contained uniform magnetic field, suffused with an isotropic distribution of relativistic electrons. The source, as it expanded, caused an evolution of the radio light curve at different frequencies. Each of the curves in Van der Laan's paper represents a factor of 2 difference 
in frequency, the vertical axis representing intensity of the radio flux and the horizontal axis representing an expansion timescale for the emitting region. Van der Laan's calculations show a marked difference between the peaks at various frequencies.

In the example of Cen A, the radio frequency bands track one another throughout the entire interval reported by Beall et al. (1978). The data from Cen A (as discussed more fully in Beall $(2008,2010)$ are, therefore, not consistent with van der Laan expansion (Van der Laan, 1966), since for van der Laan expansion, we would expect the different frequencies to achieve their maxima at different times. Even if the $10.7 \mathrm{GHz}$ data are in the synchrotron self-absorbed portion of the spectrum, as appears likely, there is no straightforward way to explain the behavior of the radio data via the van der Laan mechanism.

The observations in the hard $\mathrm{x}$-ray at $100 \mathrm{keV}$ are also problematic, since the slope of the power-law spectrum at $100 \mathrm{keV}$ hardens (i.e., flattens) at one epoch in the observations. The hard x-ray spectrum is unabsorbed, and therefore represents the spectrum of the underlying radiating electrons. Therefore, the most likely explanation for the changes in the spectrum of the Cen A data at $100 \mathrm{keV}$ (Beall, et al., 1978) is that the emitting region suffered an injection or reacceleration of energetic electrons.

A similar scenario can explain the reported radio variability. That is, the jet-ambient-medium interaction injected a population of energetic particles into a putative van der Laan "blob," or, equivalently, that there was a re-acceleration of the emitting electrons on a timescale short compared to the expansion time of the source.

An analysis of the 3C120 results compared with the data from the galactic microquasar, Sco X-1, undertaken by Beall (2006) shows a similar radio evolution, with rapidly moving "bullets" interacting with slower moving, expanding blobs. It is highly likely that the elements of these sources that are consistent with van der Laan expansion are the slower-moving, expanding blobs. I believe that the relativistically moving bullets, when they interact with these slower-moving blobs, are the genesis of the flaring that we see that seems like a re-acceleration of the emitting, relativistic particles. I note that a similar scenario could be operating in Cen A and 3C454.3.

This is not to say that the "slower-moving blobs" are not themselves moving relativistically, since the bi-polar lobes have significant enhancements in brightness due to relativistic Doppler boosting for the blobs moving toward us.

The true test of this hypothesis will require concurrent, multifrequency observations with resolutions sufficient to distinguish jet components from core emissions in galactic microquasars as well as for AGN jets.

One of the most remarkable sagas regarding the discovery of quasar-like activity in galactic sources comes from the decades long-investigation of Sco X-1 by Ed Fomalont, Barry Geldzahler, and Charlie Bradshaw (Fomalont, Geldzahler, and Bradshaw, et al. 2001). During their observations, an extended source changed relative position with respect to the primary object, disappeared, and then reappeared many times. We now know that they were observing a highly variable jet from a binary, neutron star system. The determinant observation was conducted using the Very Large Array (VLA) in Socorro, New Mexico and the VLBA interferometer (see, e.g., Beall, 2008) for a more complete discussion).

The observations of the concurrent IR, Optical, UV, and $\gamma$-ray variability of 3C454.3, and its associated jet launch in the MOJAVE data, argue for a reinvestigation of these data sets in the near 
future.

It is worthy of note that the milliarcsecond observations show a complex evolution of structure at parsec scales, including an apparently sharp change of directions associated with changes in the polarization of the radio light at that point in the jet's evolution (see Figure 2). Furthermore, the multi-frequency flares reported by Bonning et al. (2009) are consistent with the launch of another component of the astrophysical jet in the core region.

Regarding the acceleration region and the possible mechanisms for the collimation of the jets, a number of models have been proposed (see, e.g., Kundt and Gopal-Krishna (2004), BisnovatyKogan et al. (2002), Romanova and Lovelace (2009), and Bisnovaty-Kogan (2012) that might help explain the complexity present in these data.

\section{Concluding Remarks}

The data discussed therein suggest a model for the jet structures in which beams or blobs of energetic plasmas propagate outward from the central engine to interact with the ambient medium in the source region. This ambient medium in many cases comes from prior ejecta from the central source, but can also come from clouds in the Broad Line Region. The jet can apparently also excavate large regions, as is suggested by the complex structures in, for example, 3C120. The physical processes which can accelerate and entrain the ambient medium through which the jet propagates, have been discussed in detail in several venues (see, e.g., Rose et al., 1984, 1987, Beall, 1990, Beall et al., 2003, and Beall, 2010).

In this paper, we have focused on the patterns of concurrent radio and x-ray variability for Cen A, and on concurrent radio, $\gamma$-ray, optical, IR, and UV variability for 3C454.3. The data can be interpreted as being associated with a jet-launch scenario for these sources, and suggests an association between a jet launch in the MOJAVE data for 3C454.3 and its $\gamma$-ray flare from Fermi LAT. In addition, jets from microquasars show similar patterns of variability to those of AGNs. These data require us to abandon our assumptions both of spherical symmetry and of single-zone productions in our models of these sources.

The author gratefully acknowledges the support of the Office of Naval Research for this research. This research has made use of data from the MOJAVE database that is maintained by the MOJAVE team (Lister et al., 2009, AJ, 137, 3718). The author gratefully acknowledges the data provided by the MOJAVE Team for this paper.

\section{References}

[1] The Pierre Auger Collaboration, et al., 2007, Science 318, 938.

[2] Bach, U., Villata, M., Raiteri, C. M., et al. 2006, "Structure a variability in the VLBI jet of BL Lacertae during the WEBT campaigns (1995-2004)," A\&A, 456, 105.

[3] Beall, J.H. et al., 1978, Ap. J., 219, 836.

[4] Beall, J.H., Rose, W.K., 1980, Ap. J., 238, 579.

[5] Beall, J.H., 1987, Ap. J., 316, 227. 
[6] Beall, J.H., 1990, Physical Processes in Hot Cosmic Plasmas (Kluwer: Dordrecht), W. Brinkman, A. C. Fabian, \& F. Giovannelli, eds., pp. 341-355.

[7] Beall, J.H., Bednarek, W., 2002, Ap. J., 569, 343.

[8] Beall, J.H., 2002, in Multifrequency Behaviour of High Energy Cosmic Sources, F. Giovannelli \& L. Sabau-Graziati (eds.), Mem. S.A.It. 73, 379.

[9] Beall, J.H., 2003, in Multifrequency Behavior of High Energy Cosmic Sources, Chin. J. Astron. Astrophys. 3, Suppl., 373.

[10] Beall, J.H., 2009, Societa Italiana di Fisica, 98, 283-294.

[11] Beall, J.H., 2010, in Multifrequency Behaviour of High Energy Cosmic Sources, Frascati Workship 2009, F. Giovannelli \& L. Sabau-Graziati (eds.), Mem. S.A.It. 81, 395.

[12] Beall, J.H., et al., 2013, Acta Polytechnia, accepted for publication.

[13] Beall, J.H., 2011, Mem. S. A. It., 83, 283-290.

[14] Benford, Gregory, and Protheroe, R.J., 2008, M.N.R.A.S., A383, 417âĂŞ816.

[15] Bisnovatyi-Kogan, Genaddi, et al., 2002, Mem. S.A.It. 73, 1134., in Proceedings of the Vulcano Workshop on High-Energy Cosmic Sources, 2001

[16] Bisnovatyi-Kogan, Genaddi, 2013, Acta Polytechnia, accepted for publication.

[17] Bonning, E. W., Bailyn, C., Urry, C. M., Buxton, M., Fossati, G., Maraschi, L., Coppi, P.,Scalzo, R., Isler, J., Kaptur, A. 2009, Astrophys. J. Lett., 697, L81.

[18] Chaty, S., 2007, in Frontier Objects in Astrophysics and Particle Physics, F. Giovannelli \& G. Mannocchi (eds.), Italian Physical Society, Editrice Compositori, Bologna, Italy, 93, 329.

[19] Cooper et al., 2007, Ap.J. Supplement, 171, 376.

[20] Dar, Arnon, 2009, private communication.

[21] Doolin, S., and Blundell, K.M., 2009, Ap. J.,698, L23.

[22] Fomalont, E., Geldzahler, B., Bradshaw, C., 2001, Ap. J. 558, 283-301.

[23] Giovannelli, F., Sabau-Graziati, L., 2004, Space Science Reviews, 112, 1-443 (Kluwer Academic Publishers: Netherlands).

[24] Gómez et al. 2000, Science 289, 2317.

[25] Hiribayashi et al., 2000 PASJ 52, 997.

[26] Hannikainen, D.C., Rodriguez, J., 2008, in Multifrequency Behavior of High Energy Cosmic Sources, Chin. J. Astron. Astrophys. 8, Suppl., 341.

[27] Hawley, J.F., 2003, Phys. Plasmas 10, 1946/

[28] Jorstad, S.G., Marscher, A.P., Lister, M.L., Stirling, A.M., Cawthorne, T.V. et al., 2005, Astron. J. 130, 1418-1465.

[29] Jorstadt, S., Marscher, A., Stevens, J., Smith, P., Forster, J. et al., 2006, in Multifrequency Behavior of High Energy Cosmic Sources, Chin. J. Astron. Astrophys. 6, Suppl. 1, 247.

[30] Jorstad, Svetlana G., et al., 2013, Astrophysical Journal, 773, 147.

[31] Kundt, W. and Gopal-Krishna, 2004, Journal of Astrophysics and Astronomy, 25, 115-127. 
[32] Krause, M., Camenzind, M., 2003, in The Physics of Relativistic Jets in the CHANDRA and XMM Era, New Astron. Rev. 47, 573.

[33] Lightman, A. P., Eardley, D. N., 1974, Ap. J. Letters 187, L1.

[34] Lister et al., 2009, A.J., 137, 3718.

[35] Madejski, Greg M., et al., 1999, ApJ, 521, 145-154.

[36] Marscher, A.P., et al., 2002, Nature, 417, 625-627.

[37] Marscher, A.P., 2006, in Multifrequency Behavior of High Energy Cosmic Sources, Chin. J. Astron. Astrophys. 6, Suppl. 1, 262.

[38] Mioduszewski, A. J., Rupen, M. P., Walker, R. C., Schillemat, K. M., \& Taylor, G. B. 2004, BAAS, $36,967$.

[39] Mushotzky, R.F., Serlemitsos, P.J., Becker, R.H., Boldt, E.A., and Holt, S.S., Ap. J. 220, $790-797$.

[40] Paredes, J., 2007, in Frontier Objects in Astrophysics and Particle Physics, F. Giovannelli \& G. Mannocchi (eds.), Italian Physical Society, Editrice Compositori, Bologna, Italy, 93, 341.

[41] Romanova, M. and Lovelace, R., 2005, 2009, Triggering of Relativistic Jets, (Instituto de Astronomia, Universidad Nacional Autonoma de Mexico, William H. Lee and Enrico Ramirez-Ruiz, eds.); also at arXiv:0901.4753v1, astro-ph.HE, 29 Jan 2009.

[42] Rose, W.K., et al., 1984, Ap. J. 280, 550.

[43] Rose, W.K., et al., 1987, Ap. J. 314, 95.

[44] Ulrich, Marie-Helene, Maraschi, Laura, and Urry, C. Megan, 1997, Ann. Rev. Astron. Astrophys, 35, 445-502.

[45] Urry, C. Megan, 2011, J. Astrophys. Astr. 32, 139âĂŞ145

[46] van der Laan, H., 1966, Nature 211, 1131.

[47] Zanni, C., Murante, G., Bodo, G., Massaglia, S., Rossi, P., Ferrari, A., 2005 Astron. Astrophys., 429, 399.

\section{DISCUSSION}

SOLEN BALMAN How persistent are AGN jets? For example, BH (LMXRB) jets occur during outbursts in general. I thought microquasar jets are persistent in that they are always there. Some show orbital period dependencies. Is there some sort of known timesdale range that AGN soures show? That is, are jets (and then no jets) seen for a period (other than LMXB (BH) sources?

\section{JIM BEALL}

Both the microquasars and the AGN jets show persitence in their linear structures, but periodic outburst along the general jet axis. In the case of 3C454.3, for example, the jet "blobs" are emitted in a defined direction, then appear to "turn" on scales of parsecs into a direction that persists for kiloparsecs.

PETER MEINTJES Two questions: 
First, are the outflows (radio) observed fro Sco X-1 and SS 433 accompanied by a simultaneous $\mathrm{x}$-ray brightening of the sources.

Secondly, from the dip in the x-ray emission to the brightening of the source, $3 \mathrm{C} 120$, in the radio, one can constrain the velocity of the "bullets" ejected from the galaxy. Have any estimates in that regard been made?

JIM BEALL There appears to be an anti-correlation between the radio flaring and $\mathrm{x}$-ray flux in SS 433 that is similar to the pattern in $3 \mathrm{C} 120$. For AGN, the flows appear to be highly relativistic on scales of parsecs, and mildly relativistic on scales of light-weeks for microquasars.

DIMITRY BISIKALO The jet precession should be probably different for jets of different origins. Does the jet precession allow us to get some information about the different central engines?

JIM BEALL I think the usual interpretation of the precession is that it is caused by the perturbation of the accretion disk by material that is accreting in a plane off the disk equatorial plane. But the disks in these AGNs must be very large to sustain the jet axes for such a long period of time. I think this is an interesting area to focus research. 